\title{
EVALUATION OF METASTATIC STAGE PATIENT CHARACTERISTICS ATTENDED AT DR ARNALDO VIEIRA DE CARVALHO CANCER INSTITUTE
}

Fábio F. O. Rodrigues', Eveline A. Silva', Etienne A. Bastos¹, Daniel C. B. de Oliveira1', Rodrigo M. Silva1', Carlos E. Fristachi' ${ }^{1}$ Oncoginecology and Mastology Service, Dr. Arnaldo Vieira de Carvalho Cancer Institute - São Paulo (SP), Brazil.

Objectives: Evaluate the characteristics of metastatic stage patients attended at the Oncoginecology and Mastology Service (SOGM, acronym in Portuguese) of Dr. Arnaldo Vieira de Carvalho Cancer Institute (ICAVC) form 2006 to 2014, with follow-up until 2018. Methods: A descriptive, retrospective, longitudinal study was performed with patients with metastatic stage invasive breast carcinoma attended at ICAVC in São Paulo; data were analyzed on the database of Microsoft Excel ${ }^{\circledR}$. Results: 339 patients were evaluated, of which 37 (12\%) presented distant metastasis at some point in the follow-up, with a predominance of patients with tumors Stage III (32.4\%), age under 70 years (81.1\%) and luminal (83.3\%). The majority underwent radical breast surgery (78.4\%) and was not submitted to neoadjuvant chemotherapy (62.2 $\mathrm{x} 37.8 \%$ ). Of the total of 37 patients with evolution with distant metastasis, $78.4 \%$ did not present a local recurrence and, in relation to metastasis sites, the majority presented metastasis to bone (64.9\%); of these, $62.5 \%$ were luminal and only 8.3\% triple negatives. Of the 37 patients, 7 (19\%) remain alive, in outpatient follow-up; of these, $57.1 \%$ are of the luminal subtype. Conclusion: The mean follow-up time between diagnosis and the onset of the first metastasis was approximately 29 months. The luminal subtype was shown in this study as the subtype with better results in absolute numbers in terms of metastatic disease survival, showing that the subtype of breast cancer influences the results of patients. When evaluating the prevalence of the distant metastasis site, bone metastasis proved to be the most common site, which is consistent with the data in the literature. 